\title{
Microfluidic devices for examining the physical limits of migration in confined environments
}

\author{
Majid Malboubi ${ }^{1,2}$, Asier Jayo ${ }^{3}$, Maddy Parsons $^{4}$, Guillaume Charras ${ }^{2,5,6}$ \\ ${ }^{1}$ Department of Engineering Science, University of Oxford, Oxford, OX1 3PJ, UK \\ ${ }^{2}$ London Centre for Nanotechnology, University College London, London, WC1H OAH, UK \\ ${ }^{3}$ Universidad CEU San Pablo, Madrid, Spain. \\ ${ }^{4}$ Randall Division of Cell and Molecular Biophysics, Kings College London, UK \\ ${ }^{5}$ Institute for the Physics of Living Systems, University College London, London, WC1E 6BT, UK \\ ${ }^{6}$ Department of Cell and Developmental Biology, University College London, London, WC1E 6BT, UK
}

Authors for correspondence: Majid Malboubi (majid.malboubi@eng.ox.ac.uk) or Guillaume Charras (g.charras@ucl.ac.uk)

Running title: Physical limits of migration.

\section{Abstract}

Cell migration plays a key role in many physiological and pathological conditions during which cells migrate primarily in the 3D environments formed by tissues. Microfluidics enables the design of simple devices that can mimic in a highly controlled manner the geometry and dimensions of the interstices encountered by cells in the body. Here we describe the design, fabrication, and implementation of an array of channels with a range of cross-sections to investigate migration of cells and cell clusters through confined spaces. By combining this assay with a motorised microscope stage, image data can be acquired with high throughput to determine the physical limits of migration in confined environments and their biological origin.

\section{Key Words}

Microfluidics, Cell deformation, Breast cancer cells, Multilayer photolithography 


\section{Introduction}

Cell migration is involved in many aspects of development, immunity, and pathology. In embryos, neural crest cells migrate collectively from the neural tube over hundreds of microns to specific areas where they will differentiate to give rise to a number of tissues [1,2]. In response to inflammation, white blood cells exit the blood vessels across the endothelium before migrating through the tissue to the source of inflammation [3,4]. During cancer, cells migrate away from the primary tumour throughout the tissue either as groups or as individual cells [5]. Thus, although much has been learned from examining migration on planar substrates, there is a need to examine migration in three-dimensional environments and recent studies have made it abundantly clear that migration in 3D differs under many aspects from migration in 2D [6-10].

Microfabrication combined with soft lithography techniques allows the generation of microfluidic devices that can mimic the geometry and dimensions of the interstices encountered by cells during migration through tissues. In contrast to extracellular matrix protein gels that form complex and poorly ordered environments, microfluidics enables the manufacture of a simplified environment that remains identical from one experiment to the next allowing straightforward quantification and comparison between experimental conditions.

Here, we demonstrate the design, manufacture and implementation of a simple device for examining the physical limits of migration of single cells through interstices. Although the device was originally designed to examine single cell migration $[11,12]$, simple changes to the design also allow examination of the migration of cell clusters [13]. The device consists of a reservoir linked to a large channel via an array of smaller transverse channels (see Fig. 1a). After seeding the cells in the reservoir, beads soaked with chemoattractant are loaded into the large channel. Chemoattractant diffuses through the small transverse channels into the reservoir and cells migrate towards this. The device is designed such that transverse channels with many different cross-sections link the reservoir to the channel. This allows comparison of the migration of cells as a function of channel cross-section in conditions that are otherwise identical. The microfluidic chip can be assembled on a coverslip or in a glass bottom Petri dish for imaging. When used in combination with a motorised microscope stage, the device allows acquisition of large numbers of tracks per experiment. For use with cell clusters, the cross-section of the transverse channels can be increased appropriately.

Setup of the experiment involves a microfabrication step, a soft lithography step, a cell-seeding step and an imaging step. Once a master mould is fabricated in the microfabrication step, it can be used many times in the soft lithography step. This second step generates negative replica of the 
master mould using a transparent polymer to make devices for experiments. For an in depth description of the steps involved in microfluidics, we refer the interested readers to the following references $[14,15]$.

\section{Materials}

\subsection{Softwares}

1. AutoCAD for mask design

2. ImageJ for analysing images obtained during experiments

\subsection{Master-mould Fabrication}

1. 3-inch Silicon wafers

2. SU-8 2005 and SU-8 2050 photoresist (Microchem)

3. EC solvent (SU-8 Developer, Microchem)

4. Isopropanol

5. Acetate and Chrome photomasks

6. Spin coater

7. Two hot plates set to $65^{\circ} \mathrm{C}$ and $95^{\circ} \mathrm{C}$ respectively

8. Mask aligner with UV source

9. Forceps for wafer manipulation

\subsection{Soft lithography}

1. PDMS prepolymer and curing agent (Sylgard 184, Dow Corning)

2. Isopropanol

3. Acetone

4. Compressed nitrogen cylinder and nitrogen gun

5. Piranha solution

6. Petri dishes

7. Disposable plastic cups and stir rods for mixing PDMS

8. $50 \mathrm{~mm}$ glass bottom dishes

9. Scalpels

10. Biopsy punches ( $1 \mathrm{~mm}$ diameter and $4 \mathrm{~mm}$ diameter).

11. Soft tubing: Tygon non-DEHP microbore tubing, $0.010 " \times 0.030 " \mathrm{OD}, 500 \mathrm{ft} / \mathrm{roll}$

12. Hard tubing: Cole-Parmer Microbore PTFE Tubing, 0.022"ID x 0.042"OD, 100 $\mathrm{ft} / \mathrm{roll}$.

13. Blunt 25 gauge needles.

14. Trichloro(1H $1 \mathrm{H} 2 \mathrm{H} 2 \mathrm{H}$ perfluorooctyl) silane

15. Digital balance

16. Laboratory oven

17. Vacuum bell with pump

18. Plasma cleaner

19. Laminar flow hood

\subsection{Cell culture}

1. MDA MB 231 human breast carcinoma cell line (ATCC)

2. Cell culture medium: Dulbecco's modified Eagle's medium supplemented with $1 \%$ Penicillin-Streptomycin and Glutamine and 10\% v/v Fetal Bovine Serum.

3. Trypsin $0.05 \%(w / v)$ with EDTA 

4. Pipettes/tips
5. Fibronectin
6. PBS
7. $15 \mu \mathrm{m}$ diameter polystyrene beads
8. Ham's F12 medium without serum.
9. Humidified incubator with $5 \% \mathrm{CO}_{2}$
10. Centrifuge
11. Centrifuge tubes
12. Cell culture hood

3. Imaging
1. Wide-field epi-fluorescence microscope
2. Environmental control chamber
3. EMCCD camera
4. Motorised stage

\section{Methods}

\subsection{Mask design}

The design consists of two layers (see Note 1), a first thin layer containing a series of transversal channels through which the cells will migrate (see Fig. 1a, b and 1e) and a second thicker layer consisting of the open access reservoir and the large channel (see Fig. 1a and 1c). The transverse micro-channels in the first layer are $150 \mu \mathrm{m}$ long, $5 \mu \mathrm{m}$ high, and are arranged in groups with widths ranging from 2 to $20 \mu \mathrm{m}$ (see Fig. 1e). The reservoir is $16 \mathrm{~mm}$ long, $10 \mathrm{~mm}$ wide, and $80 \mu \mathrm{m}$ high. The large channel (chemoattractant channel) is $22 \mathrm{~mm}$ long, $800 \mu \mathrm{m}$ wide and $80 \mu \mathrm{m}$ high (Figure 1a). The large channel and the reservoir are separated by a gap of $100 \mu \mathrm{m}$.

1. Use AutoCAD to design the mask. All features must be drawn using 'CLOSED POLYLINES'.

2. Create a new layer, name it "Transverse microchannels", choose a colour and draw the first layer (see Note 2).

3. Create a new layer, name it "alignment marks", choose a colour and draw alignment marks (see Note 3 ).

4. Create a new layer, call it "Reservoir", choose a colour and draw the second layer (see Note 4).

5. Send the design to a mask manufacturer. The first layer needs to be printed on a chrome mask because of the small cross-section of some of the channels while the second layer can be printed on acetate because of the larger dimensions of its features (see Note 5).

\subsection{Multilayer photolithography}

Photolithography must take place in a clean-room.

1. Clean a 3-inch silicon wafer first with acetone and then with isopropanol (see Note 6).

2. Dry the wafer under a stream of nitrogen (see Note 7).

3. Pour SU-8 2005 photoresist on the silicon wafer and place in spin coater. Spin following the manufacturer protocol. After holding at 500 RPM for $30 \mathrm{~s}$, ramp to 3000 
RPM with a rate of $500 \mathrm{RPM} / \mathrm{s}$ and hold for $30 \mathrm{~s}$ to obtain a $5 \mu \mathrm{m}$ thick layer of SU-8. Check that the wafer is homogenously covered with photoresist.

4. Soft bake the wafer at $95^{\circ} \mathrm{C}$ for $2 \mathrm{~min}$ on a hot plate.

5. Affix the chrome mask containing the transverse microchannels and the alignment marks to mask aligner.

6. Place the silicon wafer coated with resist in the mask aligner.

7. Bring the silicon wafer into contact with the mask.

8. Expose the wafer through the chrome mask for $5 \mathrm{~s}$ at $20 \mathrm{~mW} / \mathrm{cm}^{2}$ to obtain a total exposure energy of $100 \mathrm{~mJ} / \mathrm{cm}^{2}$ (see Note 8).

9. Release the silicon wafer.

10. Post bake at $95^{\circ} \mathrm{C}$ for $3 \mathrm{~min}$ on the hot plate.

11. Develop in EC solvent for 1 minute in a chemical flow hood. Agitate gently.

12. Rinse with isopropanol (see Note 9).

13. Dry wafer under a stream of nitrogen.

14. Pour SU-8 2050 photoresist on the silicon wafer. Spin following the manufacturer protocol. After holding at 500 RPM for $30 \mathrm{~s}$, ramp to 1900 RPM with a rate of 500 RPM/s and hold for $30 \mathrm{~s}$ to obtain an $80 \mu \mathrm{m}$ thick layer of SU-8 (see Note 10).

15. Soft bake for $3 \mathrm{~min}$ at $65^{\circ} \mathrm{C}$ on a hot plate before moving to a $95^{\circ} \mathrm{C}$ hot plate for $9 \mathrm{~min}$.

16. Affix the acetate mask containing the cell-seeding reservoir, the chemoattractant channel and the alignment marks to mask aligner.

17. Place the silicon wafer coated with resist in the mask aligner.

18. Using the alignment marks, align the wafer with the acetate mask.

19. Bring the wafer into contact with the mask.

20. Expose for 11 seconds at $20 \mathrm{~mW} / \mathrm{cm}^{2}$ to obtain a total energy of $220 \mathrm{~mJ} / \mathrm{cm}^{2}$.

21. Release the silicon wafer.

22. Post bake for $7 \mathrm{~min}$ at $95^{\circ} \mathrm{C}$ on the hot plate.

23. Develop in EC solvent for $6 \mathrm{~min}$ in a chemical flow hood. Agitating gently throughout.

24. Rinse with isopropanol.

25. Blow nitrogen to dry the wafer (see Note 11).

26. Hard bake at $150^{\circ} \mathrm{C}$ for $6 \mathrm{~min}$.

\subsection{Soft lithography}

Soft lithography can be done in a normal laboratory. The soft lithography consists of a passivation step and a moulding step. If a master is not used for the first time, skip passivation and go directly to step 5 .

1. Place wafer in a vacuum bell.

2. Place a drop of Trichloro $(1 \mathrm{H} 1 \mathrm{H} 2 \mathrm{H} 2 \mathrm{H}$ perfluorooctyl) silane in a small glass bottom Petri dish in the vacuum bell.

3. Draw vacuum and leave to passivate for $3-4 \mathrm{~h}$. Several wafers can be passivated simultaneously.

4. Break vacuum. Dispose of silane appropriately.

5. Place the wafer in a sufficiently large petri dish.

6. Place disposable plastic cup on balance. Tare.

7. Pour PDMS primer slowly to obtain the desired weight of PDMS primer.

8. Add curing agent such that the primer to curing agent ratio is 10:1.

9. Mix PDMS with curing agent with a stirrer (see Note 12). 
10. Pour PDMS onto the mould containing the master.

11. Place in the vacuum bell. Draw vacuum and leave for $45 \mathrm{~min}$ to remove bubbles trapped around the features.

12. Break vacuum (see Note 13 ).

13. Cure at $65^{\circ} \mathrm{C}$ in oven for $1 \mathrm{~h}$.

14. Place the petri dish containing the master mould in a laminar flow hood to minimise dust particles adhering to PDMS.

15. Using a scalpel, cut around the device features leaving a gap of at least $5 \mathrm{~mm}$ (see Note 14).

16. Flip the PDMS chip over such that it is channel side up.

17. Punch holes through the PDMS at either side of the chemoattractant channel using 1 $\mathrm{mm}$ biopsy punches.

18. Punch a hole in the central part of the chamber using a $4 \mathrm{~mm}$ biopsy punch to provide an opening for cell seeding.

19. Place the PDMS chip and a $50 \mathrm{~mm}$ glass bottom dish in the chamber of plasma cleaner. Both surfaces to be treated should be facing up. Treat with oxygen plasma for $30 \mathrm{~s}$.

20. After treatment, place the PDMS chip on the glass and very gently press the PDMS chip to encourage bonding.

21. Place the assembled device into an oven at $60^{\circ} \mathrm{C}$ for $1 \mathrm{~h}$ to promote bonding.

22. Cut the hard tubing into $1 \mathrm{~cm}$ segments and push into the inlet and outlet holes of the chemoattractant channel.

23. Cut several $\mathrm{cm}$ of soft tubing. Push the soft tubes into the hard tubing as deep as possible.

24. Introduce the tip of a blunt needle through the inner diameter of the soft tubing to create an interface for a luer lock syringe.

25 . Sterilize the devices by immersing in $70 \%$ ethanol.

26. Dry in a sterile biosafety cabinet for $1 \mathrm{~h}$ before use (see Note 15).

\subsection{Cell culture, channel preparation, and cell seeding}

1. Passage MDA MB 231 human breast carcinoma cells (ATCC) as recommended by ATCC.

2. Circulate PBS through the chemoattractant channel using a syringe attached to soft tubing by the luer lock, the PBS will gently fill the cell-seeding reservoir.

3. Wash devices with PBS by gently circulating it through the channels and removing it from the cell-seeding reservoir.

4. Fill syringe with $10 \mu \mathrm{g} / \mathrm{mL}$ fibronectin in PBS and circulate through the chemoattractant channel and allow it to flow to the cell-seeding reservoir (see Note 16).

5. Incubate at $37^{\circ} \mathrm{C}$ overnight in a humidified incubator (see Note 17).

6. Wash the device three times by circulating PBS through the chemoattractant channel and drawing it from the cell-seeding reservoir.

7. Fill reservoir and chemoattractant channel with Ham's F12 medium by introducing the medium through the chemoattractant channel and drawing through the cell-seeding reservoir.

8. Trypsinise growing cells, re-suspend them in Ham's F12 medium at $5 \times 10^{6}$ cells $/ \mathrm{mL}$, and add up to $100 \mu \mathrm{L}$ of the cell suspension to the reservoir chamber using a micropipette. 
9. Leave cells to spread for $2 \mathrm{~h}$ in a humidified incubator at $37^{\circ} \mathrm{C}$.

\section{5 live cell imaging}

1. Coat $15 \mu \mathrm{m}$ diameter polystyrene beads with Fetal Bovine Serum (FBS) by incubating in Ham F12 medium with $10 \%$ FBS overnight at $4^{\circ} \mathrm{C}$.

2. Introduce beads into the chemoattractant channel with a syringe (see Note 18).

3. Place the chamber on the microscope stage for live cell imaging.

4. Allow beads to rest for $\mathbf{3 0}$ min to allow gradient formation and start live cell imaging (see Note 19).

5. Program multi-position imaging and acquire images at 10 minute intervals for $10 \mathrm{~h}$, using a 20x magnification 0.4 numerical aperture air objective (see Note 20).

\section{Notes}

1. A detailed description of the mask design and fabrication process can be found at [16] (doi:10.1038/protex.2015.069). An example of alignment marks can be found on the Stanford foundry website (https://web.stanford.edu/group/foundry/Mask\%20Design\%20Rules.html).

2. To ensure optimal bonding of the PDMS to the glass substrate, the distance between each pair of transversal micro-channels should be at least ten times the width of the larger micro-channel. The total width of a set of transversal channels is $1 \mathrm{~mm}$ enabling imaging of all channel sizes simultaneously in one field of view at $4 x$ magnification.

3. To facilitate alignment, a large overlap region between the first and the second layer should be included.

4. Because of the large overall dimensions of the cell seeding reservoir and the chemoattractant channel, pillars were included in this layer to prevent the device from collapsing. As a rule of thumb, channels with an aspect ratio larger than 1:10 (height/width) will be structurally sound. However, channels or features with aspect ratios smaller than 1:20 will collapse when fabricated with PDMS. To overcome this problem, regularly spaced pillars must be included in the design to prevent collapse.

5. The acetate mask should be printed with a resolution of 40,000 dpi.

6. For a stronger adhesion of SU-8 to the substrate, clean the wafers with piranha solution. Piranha solution is a mixture of concentrated sulfuric acid with hydrogen peroxide, usually in a ratio of 3:1 to 7:1. It is highly corrosive and an extremely powerful oxidizer and must be prepared with great care. We refer interested readers to [17] .

7. If necessary, place the wafer in an oven, or on a hot plate, for a few minutes to ensure complete dryness.

8. Exposure time $(\mathrm{s})=$ exposure energy $(\mathrm{mJ} / \mathrm{cm} 2) / \mathrm{UV}$ light power $(\mathrm{mW} / \mathrm{cm} 2)$. Check the power of the mercury lamp regularly. If the power is lower than the nominal power, recalculate the time of exposure.

9. If you observe white streaks after this step, it signifies that the development is incomplete. Return to EC solvent for longer to finish developing.

10. The thickness of this layer will define the height of the cell seeding reservoir. If the height is too small, cells may stick to the ceiling of the chamber or be squeezed in between the ceiling and the glass bottom. The height of the cell-seeding reservoir 
needs to be optimised for each cell type. Chambers with smaller heights have less nutrients available for cells. This may become an issue if long term imaging is planned.

11. Examine the wafer using a microscope to make sure that all uncrosslinked SU-8 is removed, that features are sharp, and that all channels straight. Placing the beaker containing the wafer in EC solvent for a few seconds in an ultrasonic bath may help removing uncrosslinked SU-8 from in between the transversal channels.

12. To introduce less dust cover the cup with a paraffin tape and make a few holes on it.

13. Steps 11 and 12 may need to be repeated several times until all bubbles have been removed.

14. A smaller gap may result in leakage. Do not use excessive pressure when cutting. Silicon wafers are fragile and may break. Wear gloves when handling the PDMS device and avoid touching the bottom surface containing the channels as this could compromise bonding.

15. This step must be performed in a sterile laminar flow hood to maintain sterility and minimize contamination.

16. Filling the chamber, chemoattractant channel, microchannels and reservoir by circulating medium through the chemoattractant channels has to be a gentle process to allow the exit of any air bubbles in the device.

17. This step ensures that the PDMS becomes saturated with water vapour, which enhances cell viability within devices.

18. Beads are coated with FBS to obtain a gradual delivery of chemoattractants and stable gradient formation without flow.

19. During the experiments, Petri dishes were filled with sufficient culture medium to prevent evaporation. Excess medium was also added to the Petri dish outside of the device. In some experiments, to visualise gradient formation, beads were coated with Rhodamine Isothiocyanate and FBS, washed several times, and imaged immediately after bead injection into the upper channel.

20. Use cell lines with fluorescently tagged Histone 2BK (H2BK) for simplified visualisation of the nucleus and automated cell tracking. Nuclear dyes or stains can also be used in place of H2BK. Optimise imaging interval and exposure time to minimise photodamage.

\section{References}

1. Theveneau E, Mayor R (2012) Neural crest delamination and migration: from epithelium-tomesenchyme transition to collective cell migration. Dev Biol 366 (1):34-54. doi:10.1016/j.ydbio.2011.12.041

2. Friedl $P$, Gilmour $D$ (2009) Collective cell migration in morphogenesis, regeneration and cancer. Nat Rev Mol Cell Biol 10 (7):445-457. doi:10.1038/nrm2720

3. Friedl P, Weigelin B (2008) Interstitial leukocyte migration and immune function. Nat Immunol 9 (9):960-969. doi:10.1038/ni.f.212

4. Woodfin A, Voisin MB, Nourshargh S (2010) Recent developments and complexities in neutrophil transmigration. Curr Opin Hematol 17 (1):9-17. doi:10.1097/MOH.0b013e3283333930 
5. Friedl P, Alexander S (2011) Cancer invasion and the microenvironment: plasticity and reciprocity. Cell 147 (5):992-1009. doi:10.1016/j.cell.2011.11.016

6. Wilson K, Lewalle A, Fritzsche M, Thorogate R, Duke T, Charras G (2013) Mechanisms of leading edge protrusion in interstitial migration. Nat Commun 4:2896. doi:10.1038/ncomms3896

7. Bergert M, Erzberger A, Desai RA, Aspalter IM, Oates AC, Charras G, Salbreux G, Paluch EK (2015) Force transmission during adhesion-independent migration. Nat Cell Biol 17 (4):524-529. doi:10.1038/ncb3134

8. Ruprecht V, Wieser S, Callan-Jones A, Smutny M, Morita H, Sako K, Barone V, Ritsch-Marte M, Sixt M, Voituriez R, Heisenberg CP (2015) Cortical contractility triggers a stochastic switch to fast amoeboid cell motility. Cell 160 (4):673-685. doi:10.1016/j.cell.2015.01.008

9. Liu YJ, Le Berre M, Lautenschlaeger F, Maiuri P, Callan-Jones A, Heuze M, Takaki T, Voituriez R, Piel $M$ (2015) Confinement and low adhesion induce fast amoeboid migration of slow mesenchymal cells. Cell 160 (4):659-672. doi:10.1016/j.cell.2015.01.007

10. Stroka KM, Jiang H, Chen SH, Tong Z, Wirtz D, Sun SX, Konstantopoulos K (2014) Water permeation drives tumor cell migration in confined microenvironments. Cell 157 (3):611-623. doi:10.1016/j.cell.2014.02.052

11. Malboubi M, Jayo A, Parsons M, Charras G (2015) An open access microfluidic device for the study of the physical limits of cancer cell deformation during migration in confined environments. Microelectron Eng 144:42-45. doi:10.1016/j.mee.2015.02.022

12. Jayo A, Malboubi M, Antoku S, Chang W, Ortiz-Zapater E, Groen C, Pfisterer K, Tootle T, Charras G, Gundersen GG, Parsons M (2016) Fascin Regulates Nuclear Movement and Deformation in Migrating Cells. Dev Cell 38 (4):371-383. doi:10.1016/j.devcel.2016.07.021

13. Kuriyama S, Theveneau E, Benedetto A, Parsons M, Tanaka M, Charras G, Kabla A, Mayor R (2014) In vivo collective cell migration requires an LPAR2-dependent increase in tissue fluidity. J Cell Biol 206 (1):113-127. doi:10.1083/jcb.201402093

14. Tabeling P (2010) Introduction to Microfluidics. Oxford University Press, Oxford 15. Folch A (2012) Introduction to BioMEMs. CRC Press, Boca Raton, FI

16. Lake M, Narciso C, Cowdrick K, Storey T, Zhang S, Zartman J, Hoelzle D (2015) Microfluidic device design, fabrication, and testing protocols. Protocol Exchange. doi:doi:10.1038/protex.2015.069

17. Millet LJ, Stewart ME, Sweedler JV, Nuzzo RG, Gillette MU (2007) Microfluidic devices for culturing primary mammalian neurons at low densities. Lab Chip 7 (8):987-994. doi:10.1039/b705266a 

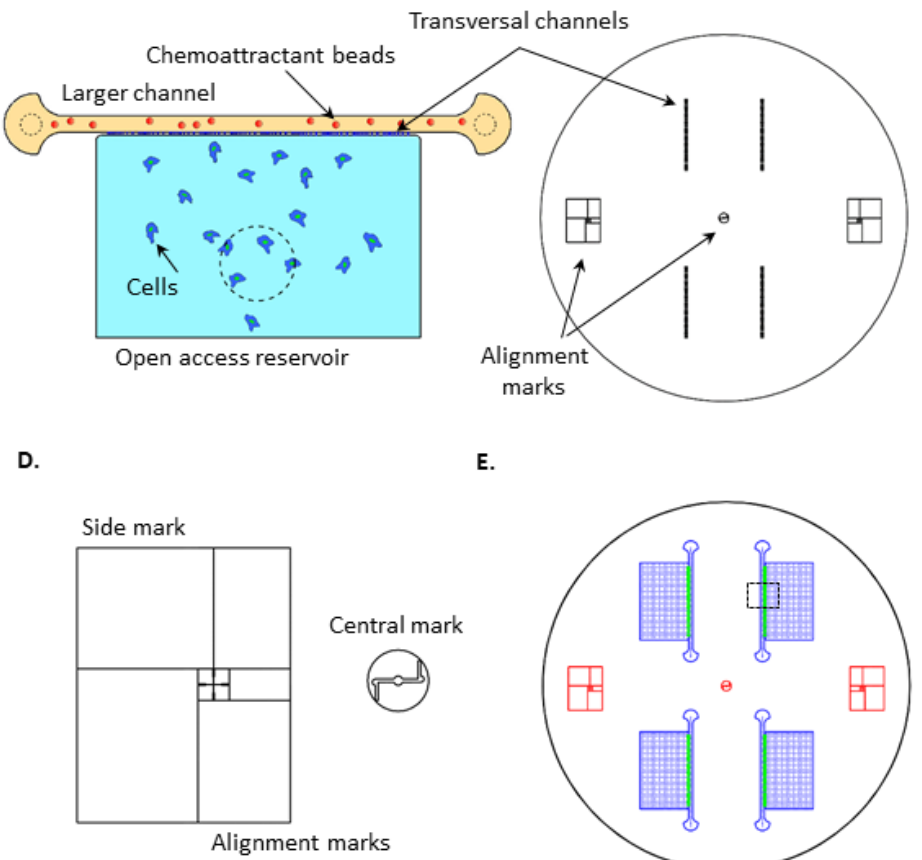

B.

E.

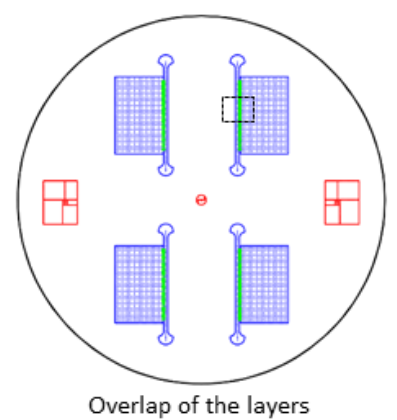

c.

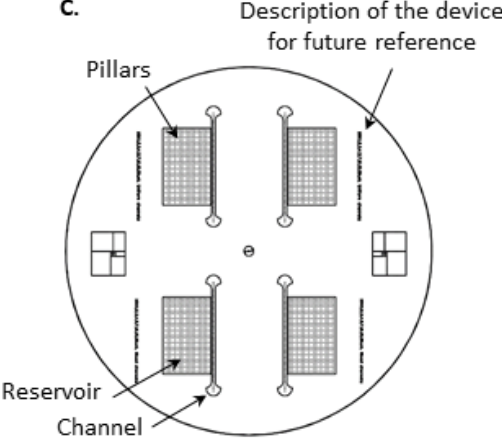

Transversal channels from 2 to $20 \mu \mathrm{m}$ width

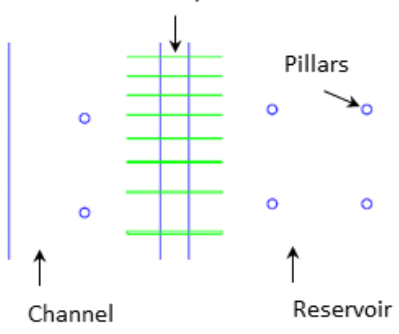

Figure 1. Device design. A. Schematic design of the device. B. Layer 1, Transverse channels. C. Layer 2, Open access reservoir and the channel for circulating chemoattractant. D. Alignment marks. E. Overlap of two layers. Layer 1 is shown in green. Layer 2 is shown in blue. Alignment marks in red. F. Detailed view of transverse channels connecting the reservoir to the channel. This region is a zoomed view of the boxed region in $\mathrm{E}$. 


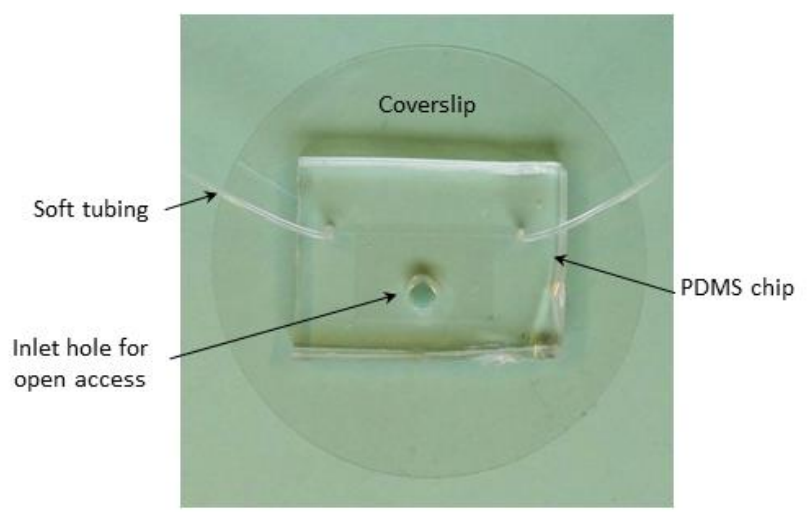

Figure 2. Completed device. 
A.
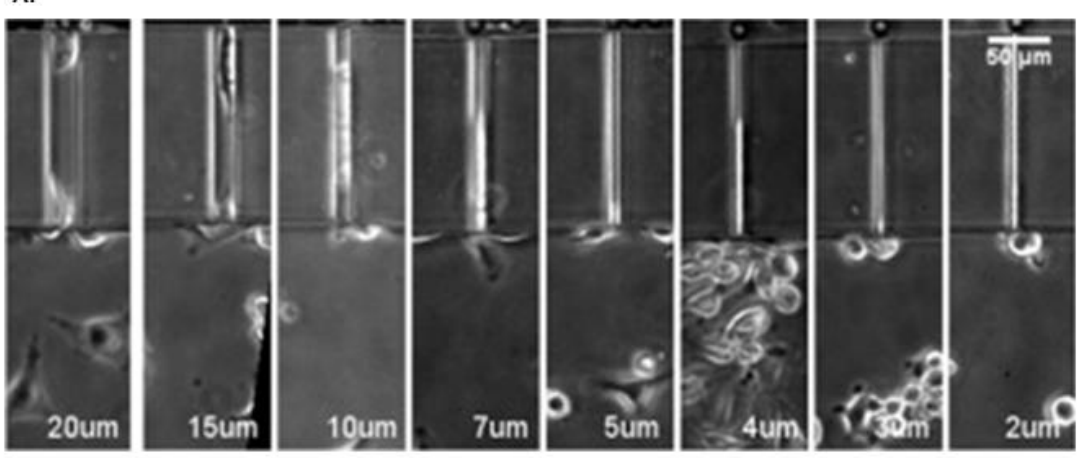

$$
\text { B. }
$$
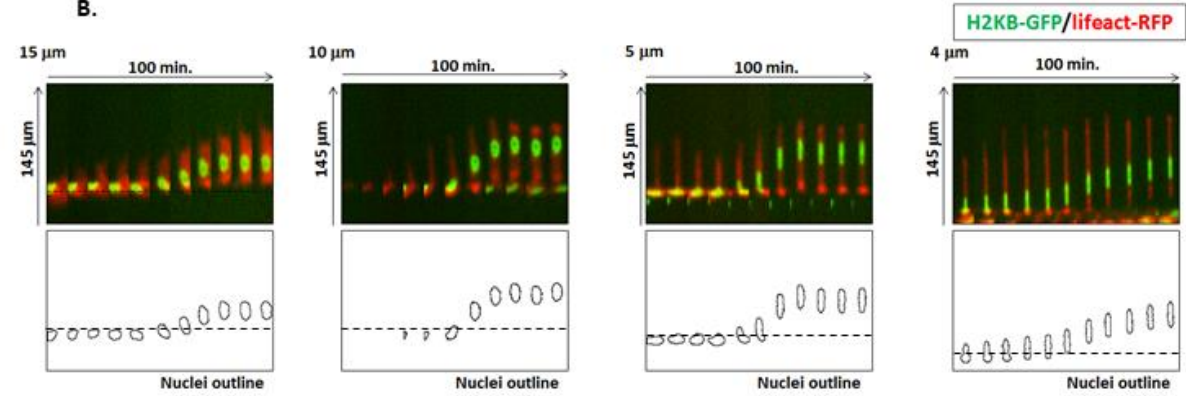

Figure 3. A. Phase contrast image of the channels from [11]. B. Kymographs of representative single cells translocating their nuclei into channels of different width. The upper panel shows cells expressing GFP-H2BK (green) and RFP-lifeact (red). The lower panel shows the outline of the nuclei. Dashed lines indicate the entrance to the channels. The width of each channel is indicated in the top left above the fluorescence images. 